\title{
Essais
}

Revue interdisciplinaire d'Humanités

\section{Aux marges du système verbal}

Réflexions sur la valeur expressive des formes verbales surcomposées en allemand et en français dans le langage parlé

Norman $\mathrm{Haß}$

\section{(2) OpenEdition}

1 Journals

Édition électronique

URL : http://journals.openedition.org/essais/8249

DOI : 10.4000 /essais.8249

ISSN : 2276-0970

Éditeur

École doctorale Montaigne Humanités

Édition imprimée

Date de publication : 15 juillet 2014

Pagination : 90-108

ISBN : 978-2-9544269-3-8

ISSN : 2417-4211

Référence électronique

Norman Haß, «Aux marges du système verbal », Essais [En ligne], 5 | 2014, mis en ligne le 13 avril 2021, consulté le 15 avril 2021. URL : http://journals.openedition.org/essais/8249 ; DOl : https:// doi.org/10.4000/essais.8249 


\title{
Aux marges du système verbal Réflexions sur la valeur expressive des formes verbales surcomposées en allemand et en français dans le langage parlé
}

\author{
Norman Hass
}

\section{Introduction : Les formes verbales surcomposées en français et en allemand}

Les formes verbales surcomposées en français

Les formes verbales surcomposées sont le résultat d'une double application de la stratégie qui permet de former un passé composé (ou bien un plusque-parfait). ${ }^{1}$ Il s'agit des constructions du type : "Ce couteau a eu coupé ». La linguistique les appelle "formes surcomposées ", formes que la plupart des francophones prétendent ne pas connaître du tout et surtout ne jamais utiliser. Dans la littérature linguistique - pourtant vaste en ce qui concerne les temps verbaux mais assez limitée à l'égard des formes surcomposées, elles ont été traitées pendant longtemps comme un phénomène marginal, nonstandard, régional et redondant du langage parlé, et l'accent a souvent été mis sur leur caractère interchangeable par des formes dites « régulières » - surtout le passé antérieur ou bien le passé composé "simple ».2

Pour le français, on postule avec Régnier (1974) l'existence de quatre types d'emploi qui forment deux sous-groupes selon leur rapport avec le moment de l'énonciation. ${ }^{3}$

1 Cf. Amman Andreas, "The fate of "redundant" verbal forms-Double perfect constructions in the languages of Europe ». In Stolz Thomas (éd.), Sprachtypologie und Universalienforschunganguage Typology and Universals. Berlin, 2007, p. 186-204, ici p. 186.

2 Cf. p. ex. Foulet Lucien, "Le développement des formes surcomposées ", in Romania 51, Paris, 1925, p. 203-253 ; Litvinov Victor P., Radcenko Vladimir I., Doppelte Perfektbildungen in der deutschen Literartursprache. Tübingen, 1998 ; Thieroff Rolf, Das finite Verb im Deutschen Tempus-Modus-Distanz. Thèse de doctorat (Freie Universität Berlin), Tübingen, 1992 ; Stefanini Jean, «La tradition grammaticale française et les temps surcomposées », in Annales de la Faculté des Lettres d'Aix, nº 28, 1954, p. 67-108.

3 Cf. Régnier Claude, "Passé simple, passé composé, passé surcomposé dans les parlers du Morvan ", in Caluwé Jacques de et al. (éd.), Mélanges d'histoire littéraire, de linguistique et de 
Voici les quatre types tels qu’ils sont répertoriés dans la littérature scientifique :

Emploi 1: Quand il a eu fini, il est parti.

Emploi 2 : Il a eu vite fait.

Emploi 3 : (Du blé), j’en ai eu récolté du plus beau.

Emploi 4 : (Du chanvre), on en a eu fait.

Les deux premiers types d'emploi sont considérés comme standard. Leur utilisation n'est pas confinée à une région spécifique de la francophonie. C'est tout le contraire pour les deux derniers types qui sont limités au Midi de la France et à l'aire franco-provençale. ${ }^{4}$ L'emploi du type 1 est associé à des contextes grammaticaux spécifiques. On l'observe dans des phrases subordonnées, introduites par des conjonctions comme quand, lorsque, dès que, aussitôt que, après que etc. Ici, le surcomposé désigne presque toujours l'état consécutif au procès dénoté par le verbe principal de la subordonnée. Par conséquent, dans ces contextes grammaticaux, le passé surcomposé et le passé composé peuvent être remplacés respectivement par un passé antérieur et un passé simple, et cela sans modifier les conditions de vérité. ${ }^{5}$

Quand il a eu fini, il est parti.

= Quand il eut fini, il partit.

L'emploi de type 2, plus rare que l'emploi du type 1, apparaît dans des propositions indépendantes contenant un adverbial comme vite ou aussitôt. L'effet sémantique est celui d'une " précipitation " de l'action. ${ }^{6}$ Ce que les deux premiers types, qui correspondent à ce que Cornu (1953) appelle le "sens fondamental " ${ }^{7}$, ont en commun est le fait qu'il n'y a aucun rapport entre l'éventualité elle-même et le moment d'énonciation. ${ }^{8}$ Cette caractéristique les distingue de l'emploi des types 3 et 4 où le passé surcomposé véhicule une certaine "pertinence actuelle " ${ }^{9}$ de l'éventualité. Puisque ce sont ces deux derniers types d'emploi, dit le passé surcomposé régional, qui concernent le présent article, regardons tout d'abord comment fonctionne cette pertinence au moment de la parole. Une occurrence, trouvée dans le corpus du français parlé CORPAIX (Aix en Provence) nous servira d'illustration.

philologie romanes offerts à Charles Rostaing par ses collègues, ses élèves et ses amis. Association des romanistes de l'université de Liège, 1974, p. 855-870, ici p. 866.

4 Cf. Apothèloz Denis, "La quasi-synonymie du passé composé et du passé surcomposé dit "régional" ", in Pratiques : théorie, pratique, pédagogie, n 141-142, 2009, p. 98-120, ici p. 101.

5 Cf. Schaden Gerhard, La sémantique du parfait. Études des temps "composés" dans un choix de langues germaniques et romanes. Thèse de doctorat, Université Paris 8, p. 191.

6 Cf. Apothèloz, 2009, p. 101.

7 Cf. Cornu Maurice, Les formes surcomposées en français. Bern, Francke, 1953.

8 Cf. Schaden Gerhard, 2007, p. 191.

9 Cf. ibid. 


\section{Exemple 1 - CORPAIX :}

«Ben il y a une chose que j'ai trouvée énormément changée. C'est que les gens quand même mettent beaucoup moins d'argent dans leur décoration. Il y a vingt ans en arrière, quand j'ai commencé à travailler, les tissus étaient plus chers en rapport. Et les gens n'hésitaient pas à faire de très très beaux rideaux. Euh j'ai eu vendu du tissu, euh, il y a vingt plus de vingt ans en arrière qui coutait par exemple neuf cent francs le mètre..."

Dans cet exemple, nous trouvons la combinaison typique de valeurs sémantiques de cette forme verbale. Il s'agit d'un événement définitivement accompli et dont il est donc très improbable qu'il se reproduise, inscrit dans un passé lointain avec une localisation temporelle indéfinie. ${ }^{10}$ Il ne serait donc pas possible de continuer cette phrase en disant : $\left({ }^{*}\right)$ J'ai eu vendu du tissu et je continue à le faire. Mais comment peut-on caractériser cette fameuse pertinence au moment de l'énonciation ? Dans notre exemple, une vendeuse se plaint du fait que les gens ne veulent plus dépenser beaucoup d'argent pour leur décoration. Le présent du verbe "mettre " nous indique qu'elle parle de la situation actuelle qui est quand même très différente de celle d'il y a plus de vingt ans et qui n'existe plus. Le tableau suivant visualise ce constat :

\section{Intervalle de validation}

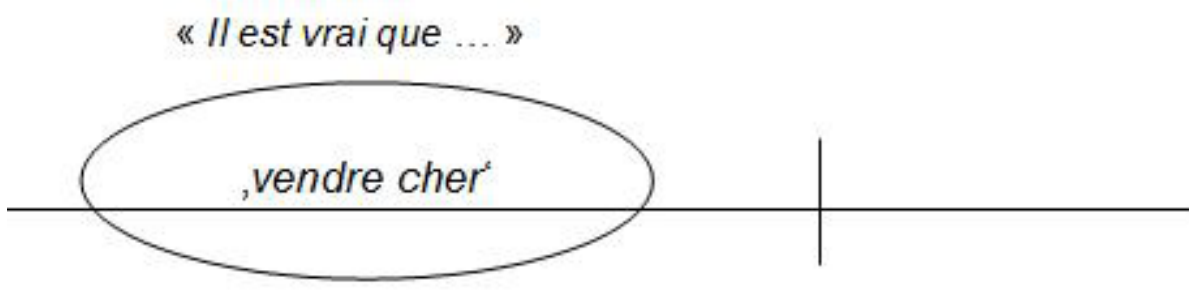

\section{Moment d'énonciation}

\section{"Maintenant »}

Il nous montre qu'un événement a eu lieu dans le passé mais n'est plus valable au moment de l'énonciation. Ce ne peut donc pas être l'événement lui-même qui est pertinent au moment de la parole. Ce que nous observons dans ces exemples, c'est que l'existence d'une situation passée est comparée avec ce qui peut être observé dans le présent et que cette mise en contraste a pour but de faire savoir que la situation, qui était valable dans le passé, n'existe plus dans le présent. ${ }^{11}$ Souvent, un connecteur comme mais est utilisé pour

10 Cf. Carruthers Janice, "The Passé Surcomposé Régional: Towards a Definition of its Function in Contemporary Spoken French ", in Journal of French Language Studies, 4:2, 1994, p. 171-190, ici p. 176.

11 Cf. Apothèloz Denis, 2009, p. 115. 
nier explicitement l'éventualité pour le moment de l'énonciation. La référence au moment de l'énonciation consiste donc à mettre en contraste le passé avec le présent.

Avons-nous donc trouvé une valeur propre aux seules formes surcomposées ? Rien n'est moins sûr. Considérons l'exemple suivant rencontré sur Internet.

Exemple 2:

"Un musicien doit être complètement sincère. Autrefois, j’ai pu jouer deux ou trois choses que je n'aimais pas beaucoup, mais je ne le fais plus depuis vingt ans. "

Comme dans le premier exemple, nous mettons ici en contraste une situation qui n'existe plus depuis longtemps, à savoir la capacité du locuteur à jouer des pièces qu'il n'aime plus, chose dont il n'est plus capable depuis vingt ans. La différence est la suivante : la comparaison avec le présent est construite sur un passé composé simple et non pas un surcomposé. Faut-il alors parler d'une synonymie entre le passé composé et le surcomposé ? Ce que nous montrent des exemples comme celui-ci, c'est que le passé composé en tant qu' " hybride sémantique " est tout à fait capable d'exprimer toutes les caractéristiques sémantiques du passé surcomposé. ${ }^{12}$ D'ailleurs, dans les corpus dont nous disposons, la vaste majorité d'énonciations qui font référence à un passé lointain est au passé composé. Ceci est un indicateur fort qui nous montre que la vraie raison de l'emploi d'un surcomposé à la place d'un composé simple doit être ailleurs.

Nous pouvons donc, à ce stade, résumer ainsi nos résultats : le passé surcomposé régional français réunit une combinaison de caractéristiques temporelles et aspectuelles, notamment la localisation de l'éventualité dans un passé (lointain), la présentation de l'action (procès, activité...) comme accomplie et le caractère indéfini de l'action. On a vu aussi qu'il partage toutes ces caractéristiques avec le passé composé simple.

\section{Les formes verbales surcomposées en allemand}

Comme pour le passé composé français, l'allemand dispose d'une forme verbale composée, le Perfekt, formé avec l'auxiliaire haben ou sein, (avoir ou être) et le Partizip II du verbe principal. Comme en français, la reprise de l'auxiliaire sous forme de participe passé donne naissance à une forme surcomposée, un double Perfekt ou un double Plusquamperfekt (plus-que-parfait) du type : "Das habe ich dir doch gesagt gehabt. " ("Mais enfin je te l'ai dit. ") Existant dans tous les dialectes allemands, ces formes sont fortement stigmatisées. Pour l'allemand tout comme pour le français, les analyses des formes surcomposées 
se divisent grosso modo en deux grandes tendances : les analyses temporelles et les analyses aspectuelles. Ainsi certains chercheurs ont déterminé leur valeur en leur attribuant la capacité de faire référence à un événement qui se situe dans le passé avant un autre événement lui-même déjà dans le passé (antérieur d'un antérieur) ou bien en mettant en avant leur valeur aspectuelle " accompli " ou "perfectif ". Dans ce qui suit, nous montrerons brièvement que les deux interprétations peuvent expliquer bon nombre d'utilisations des formes surcomposées mais que les deux ont en même temps bien des limites.

En ce qui concerne l'interprétation du double Perfekt en tant qu'antérieur d'un antérieur, c'est la prétendue origine linguistique de ces formes dans la dérive prétéritale qui semble conforter cette hypothèse. ${ }^{13} \mathrm{Il}$ s'agit du processus de la perte du Präteritum, d'abord dans le sud de l'espace germanophone, plus précisément dans les régions où l'on parle les dialectes haut-allemands. Progressivement, le passé composé allemand a évincé le prétérit dans le langage parlé dans une grande partie de l'espace germanophone. Cela a des conséquences pour une autre forme verbale, auparavant formée avec le Präteritum de l'auxiliaire, à savoir le Plusquamperfekt. L'apparition fréquente des formes surcomposées dans le sud a été expliquée très tôt comme une tentative de remplacer le Plusquamperfekt perdu, et il y a bien sûr des cas où cela semble tout à fait plausible. Comme le montre l'exemple suivant, le double Perfekt peut indiquer une antériorité. ${ }^{14}$

Exemple 4 - Dialecte : bas-allemand (nord), Basse-Saxe (nord) ; le locuteur utilise le «Plusquamperfekt » :

« $\mathrm{Na}$ ja, so um acht, halb neun, neun, genau weiß ich das nun auch nicht mehr, sind wir dann (PAUSE) da haben wir des Morgens erst mal was gegessen gehabt, und dann sind wir dann um neun Uhr losgefahren wieder. »

«Alors, vers huit heures, huit heures et demie, neuf heures, je ne me rappelle plus exactement, nous sommes (pause), d'abord nous avions mangé quelque chose le matin et après, à neuf heures nous sommes repartis. "

Dahaben wir des Morgens erstmal was gegessen gehabt. Avons nous le matin d'abord quelquechose mangé eu.

En revanche, les formes surcomposées étant très tôt apparues dans le nord de l'Allemagne où le prétérit et donc le plus-que-parfait étaient encore intacts, et d'autre part le fait que le plus-que parfait n'ait pas complètement disparu en Allemagne du Sud devrait suffire à démontrer que le rapport entre la perte d'une forme verbale et l'apparition d'une autre ne peut pas être aussi direct

13 Cf. par ex. « Duden-Grammatik, Duden. Die Grammatik, 7, völlig neu erarbeitet und erweiterte Auflage », 2005.

14 Sauf précision explicite, tous les exemples de l'allemand parlé sont issus du corpus Datenbank «Gesprochenes Deutsch » de l'" Institut für deutsche Sprache » (IdS Mannheim). Voir annexe pour la liste complète des occurrences en allemand. 
que ça. Notre dernier exemple montre que le double Perfekt peut même être combiné avec des adverbiaux indiquant une suite au sein d'une narration, indice de plus remettant en question une interprétation des surcomposés allemands en tant que simple substitut au Plusquamperfekt.

Exemple 5 - Dialecte : bas allemand (est), Poméranien (est) ; le locuteur utilise le "Plusquamperfekt» :

"Aber Mutter, die wollte das doch nicht so ganz glauben, ich lag schon im Bett. Und dann nachher am nächsten Tag, dann hat sie mich natürlich vorgekriegt gehabt, hat gesagt: „Junge, willst du es jetzt noch bestreiten?" "

"Mais ma mère ne voulait pas le croire, moi, j'étais au lit. Et puis, après, le lendemain, elle m'a bien-sûr interrogé, elle a dit : Dis-moi, mon fils, veux-tu toujours le nier?"

Und dann nachher, am nächsten Tag, dann hat sie mich natürlich vorgekriegt gehabt.
Et puis, après,
le lendemain
a elle me bien sûr interrogé
eu.

Ceci a amené les chercheurs à explorer une autre piste à savoir celle de l'aspectualité. C'est notamment le cas de Rödel (2006) ${ }^{15}$. Dans sa thèse, l'auteur essaie de démontrer que le passé surcomposé en allemand sert à grammaticaliser l'aspect perfectif dans une langue qui ne force pas le locuteur à se restreindre à un aspect particulier, contrairement au français. Comme pour la théorie du substitut au plus-que-parfait, il y a bien sûr de bons arguments pour cela.

Exemple 6 - Dialecte : alémanique, alémanique (nord) ; le locuteur n'utilise pas le "Plusquamperfekt » :

"(...) und dann sind wieder alle Bohnenkerne weg gewesen, die Vögel und

Tauben haben sie alle gefressen gehabt (...)"

"(...) puis, tous les haricots secs étaient disparus, les oiseaux et les pigeons les avaient tous mangés (...)»

Die Vögel und Tauben haben sie alle gefressen gehabt.

Les oiseaux et lespigeons ont les tous mangés eu.

Dans cet exemple, où le locuteur se souvient d'un conte de fées de son enfance, l'événement " manger tous les haricots secs " sert, tel un résultatif, à expliquer l'état qui résulte de l'événement lui-même, à savoir l'absence de tous les haricots secs. D’après Rödel (2006), les formes surcomposées en allemand permettent d'accentuer les informations qui, à travers les deux participes gehabt ou gewesen, ont été "implémentées » dans la construction qui contenait d'abord le seul Perfekt. ${ }^{16}$ Le plus important est le type d'information auquel il pense. Pour Rödel (2006), c'est une mise en relief de l'accomplissement de la situation,

15 Rödel Michael, Doppelte Perfektbildungen und die Organisation von Tempus im Deutschen. Thèse doctorat (=Studien zur deutschen Grammatik 74), 2007.

16 Cf. Rödel Michael, 2007, p. 201. 
un effet qu'on observe d'après lui surtout en langage parlé. Il concède qu'il s'agit d'un emploi qui n'est pas nécessaire pour assurer la grammaticalité du propos. En ce sens, l'utilisation de ces formes serait « en quelque sorte redondante ».

Plusieurs aspects de cette interprétation nous semblent problématiques. D'abord, comme nous le verrons plus tard, il y a des occurrences où le procès n'est pas présenté comme étant accompli. Deuxièmement, comment expliquer l'apparition de ces formes rares dans des narrations au passé dans lesquelles tous les autres événements racontés au Perfekt sont accomplis de la même façon que celui raconté au double Perfekt? Et finalement, si le Partizip II de l'auxiliaire de la forme surcomposée n'apportait rien d'autre à la construction que la mise en relief de l'accomplissement de la situation, et si son but était vraiment de souligner cet accomplissement, ne pourrait-on pas s'attendre à ce que ce Partizip II porte un accent contrastif ? Or, pour la grande majorité des exemples du corpus " Gesprochenes Deutsch » ( Langage Parlé Allemand »), qui permet également de consulter l'enregistrement des propos des locuteurs, ceci n'est pas le cas. En revanche, s'il y a un accent contrastif, il est mis sur le Partizip du verbe principal, ou bien, comme nous le verrons, on constate une intonation marquée de toute la phrase contenant la forme surcomposée. Dans ce qui suit nous essayerons de montrer que le surcomposé allemand dispose, comme son équivalent français, d'une forte valeur expressive.

\section{Les formes surcomposées en français et en allemand et la fonction expressive}

Dans cette partie, nous examinerons exclusivement des exemples issus des corpus du langage parlé pour les deux langues car nous pensons que c'est ici que s'exprime l'une des principales fonctions qui les distinguent des formes composées simples. Il s'agit de l'expressivité telle qu'elle se réalise dans des conditions qui sont propres au langage parlé. Ses conditions de communication et les stratégies de communication qui en résultent ont été décrites par différents auteurs. Nous suivrons ici le modèle de communication de Koch/ Oesterreicher (Nähe-Distanz-Modell).$^{17}$ D'après ces auteurs, le discours à proximité se caractérise entre autres par l'implication personnelle des locuteurs, la spontanéité et le lien étroit avec la situation de communication de, ce qui l'oppose au discours à distance. Dans le cas du dernier, qui est typique de la communication écrite, la situation est marquée par la réflexion, l'éloignement de la situation communicative et l'objectivité. Ces conditions différentes provoquent des stratégies communicatives différentes. Nous essayerons de montrer que les formes surcomposées peuvent être considérées dans le discours à proximité comme une stratégie expressive de mise en relief.

17 Cité d'après Hennig Mathilde, Grammatik der gesprochenen Sprache in Theorie und Praxis. Kassel, 2006, p. 69. 
L'idée qu'un fait raconté au passé surcomposé décrirait un événement hors du commun pour le locuteur a déjà été évoquée dans la littérature scientifique. ${ }^{18}$ Elle conjugue la notion d'une grande importance de l'événement pour le locuteur à l'implication personnelle. Cette interprétation nous renvoie au concept linguistique de l'expressivité. Comme le montrent des notions comme "grande importance » et "implication personnelle ", il s'agit d'un concept qui, contrairement à d'autres comme "temps " ou " aspectualité ", est plus difficile à définir. Laurent Gautier constate à juste titre que "ce qui est passionnant dans la question de l'expressivité, c'est justement son imprécision ". ${ }^{19}$ Pour notre analyse, nous nous appuierons sur la définition du Dictionnaire de linguistique et des sciences du langage (2012) :

«1. On appelle fonction expressive la fonction du langage par laquelle le message est centré sur le locuteur, dont il exprime les sentiments. 2. On appelle trait expressif un moyen syntaxique, morphologique, prosodique qui permet de mettre une emphase sur une partie de l'énoncé et suggère une attitude émotionnelle du locuteur. $»^{20}$

Les chercheurs travaillant sur l'expressivité semblent d'être d'accord sur le fait que cette emphase s'exprime grâce à un contraste entre des moyens linguistiques neutres et marqués. Dans notre cas, elle serait le résultat du contraste entre la forme verbale composée simple et la forme verbale surcomposée. Notre hypothèse est que ces formes marquées disposent de la capacité de mettre en relief une action verbale en suggérant que cette dernière est vraie plutôt que fausse, une valeur qu'on pourrait nommer "vérité ». Ainsi l'une des fonctions qui distinguent les formes surcomposées des formes non-marquées serait d'exprimer qu'une action verbale «s'est vraiment déroulée comme cela ».

\section{Les données du français}

Pour commencer, regardons cet exemple français où la personne parle de changements de vie dans la ferme.

Exemple $3:{ }^{21}$

"Mon père en a vu plus que nous d'accord mais bon j’ai aussi eu travaillé avec le cheval."

Ce qui est particulièrement intéressant ici c'est que l'événement raconté au passé surcomposé est postérieur à l'événement au passé composé. (Le travail du père avec des chevaux précédant celui du fils.) Comme nous le montre

18 Cf. p. ex. Cohen Marcel, Toujours des regards sur la langue française. Paris, 1970, p. 165.

19 Cf. Gautier Laurent, Monneret Philippe, " Présentation ", in Gautier Laurent, Monneret Philippe (éd.), La fonction expressive, vol. 2. Toulouse, 2010, p. 9-18, ici p. 9.

20 Cf. Dubois Jean et al., Le dictionnaire de linguistique et des sciences du langage. Paris, 2012, ici p. 191.

21 Exemple emprunté à Janice Carruthers, 1994, p. 182. 
Carruthers (1994), ce n'est probablement pas le fait que l'événement a eu lieu dans un passé lointain qui a amené le locuteur à utiliser le surcomposé. Il n'est donc pas très probable que le choix du surcomposé se justifie par une raison temporelle ou aspectuelle. C'est plutôt la nature exceptionnelle que le locuteur veut souligner en utilisant un surcomposé, ici, le fait que même le fils a encore connu le travail avec le cheval. La forme verbale plus complexe, lourde et phonétiquement marquée (le fait que le " eu " peut porter un accent a souvent été évoqué2 ${ }^{2}$ ) contraste avec la forme simple du passé composé. Elle attire l'attention du locuteur à un aspect inattendu un peu comme le font aussi d'autres moyens d'expressivité, par exemple la linéarisation d'informations dans une phrase (Cf. Gautier, 2007). ${ }^{23}$

\section{L'expressivité de formes surcomposées en allemand}

En allemand nous constaterons des effets comparables à celui de l'exemple français cité plus haut. Mais à notre avis, la raison n'en est pas seulement le fait que la forme est marquée. Nous voyons des parallèles avec le concept du "Verum Focus " tel qu'il est utilisé par exemple par Renate Musan. ${ }^{24}$ D'après elle, ce serait ici l'une des contributions de l'auxiliaire (haben ou sein) dans le Perfekt (simple) allemand. Cette contribution, Musan la rend visible en mettant un accent contrastif ("focus accent») sur l'élément en question. Pour l'allemand, l'exemple suivant (exemple construit) semble montrer que le participe II de l'auxiliaire porte lui aussi une valeur de "vérité ». Elle est éventuellement même plus prononcée que celle que porte l'auxiliaire simple.

Warum haben Sie ihren Mann denn geheiratet, wenn Sie ihn gar nicht lieben? Damals habe ich ihn doch geliebt gehabt!

Pourquoi avez-vous épousé votre mari puisque vous ne l'aimez pas? Mais à l'époque je l'aimais!

Ici, le " gehabt ", qui porterait très probablement même l'accent de la phrase, semble souligner (accompagné de la particule modale " doch ») la vérité de l'existence de l'amour dans le passé. En français, dans le même contexte, on n’utiliserait pas un passé surcomposé :

*Mais à l'époque, je l'ai eu aimé.

22 Cf. p. ex. Christmann Hans Helmut, "Zu den Formes Surcomposées im Französischen », in Zeitschrift für französische Sprache und Literatur, Bd. 68, H 1/2, 1958, p. 72-100, ici p. 80.

23 Cf. Gautier Laurent, "Linéarisation et expressivité dans un type de texte spécialisé : le compte rendu boursier ", in Paulin Catherine (éd.), La fonction expressive, vol. 1. Paris, 2007, p. 39-52, ici p. 49.

24 Cf. Musan Renate, "The Present Perfect in German: Outline of its Semantic Composition ", in Natural Language and Linguistic Theory, 19. Springer Netherlands, 2001, p. 335-401, ici p. 380 . 
Faut-il en déduire que le " eu " ne dispose pas de valeur de "vérité " et serait juste une marque de passé ? À ce stade de nos recherches, nous n’avons pas encore la réponse à cette question. En revanche, pour l'allemand, les exemples suivants semblent conforter notre hypothèse.

\section{La même situation exprimée en Perfekt et en double Perfekt}

Les cas où le locuteur utilise deux fois les mêmes propos sont particulièrement intéressants, une fois avec une forme composée simple, c'est-à-dire le Perfekt, et une fois avec le double Perfekt. Dans tous les exemples, c'est la forme composée simple qui précède la forme surcomposée, cette dernière apparaissant tantôt dans la phrase suivante, tantôt un peu plus loin. L'effet de cette reprise, semble-t-il, est toujours le même : la mise en relief de cet événement.

Exemple 7 - Dialecte : bas-allemand (nord), Holstein ; le locuteur utilise le « Plusquamperfekt »:

S1: Haben Sie dann auch mal ein Kinderfest in der Schule so mitgemacht? S2: Ja, Kinderfest habe ich ja in mein ersten Jahren ja mitgemacht, ${ }^{(1)}$ weil das nachher in den Kriegsjahren wurde das ja abgesagt, da waren ja keine öffentliche Veranstaltungen irgendwie. In, in den ersten Jahren habe ich das ja mitgemacht gehabt..$^{(2)}$ Dann war ja Topfschlagen und Fischstechen und Eierlaufen, alles so was mußte man ja, nicht, und.

S1 : Est-ce que vous avez aussi participé aux fêtes pour les enfants à l'école ? S2 : Oui, les fêtes d'enfants, j’y ai participé dans mes premières années ${ }^{(1)}$ parce qu'après, pendant les années de guerre, ça a été annulé, il n'y avait plus de manifestations publiques. Dans, dans les premières années j'y ai participé. ${ }^{(2)} \mathrm{Il}$ y avait " taper sur la casserole ", " course d'œufs " (jeux d'enfants) tout ce qu'il fallait...

(1) Ja, Kinderfest habe ich ja in meinen ersten Jahren ja mitgemacht. Oui, fêtes pourles enfants ai je dans mespremières années participé.

(2) In, in den ersten Jahren habe ich das ja mitgemacht gehabt. Dans, dans les premières années ai je y participé eu.

Dans cet exemple, c'est le fait d'avoir participé aux fêtes pour les enfants qui est mentionné deux fois. L'utilisation du même adverbe de temps « dans mes/ les premières années " montre bien qu'il s'agit d'un événement dans le passé qui est confiné à une certaine période, à savoir les années avant le début de la guerre. La première occurrence permettrait-elle une lecture imperfective de l'action, c'est-à-dire envisagée dans sa durée, tandis que la deuxième soulignerait l'accomplissement ? À notre avis, ceci n'est pas la lecture privilégiée de la première occurrence, le focus étant plutôt sur la participation en tant que telle. De surcroît, c'est la deuxième occurrence au surcomposé qui déclenche par la suite une représentation plus détaillée de ces fêtes pour les enfants à savoir les jeux auxquels les enfants ont joué. Comment interpréter cette observation ? Il nous semble peu probable que ce soit le souhait de mettre en avant l'accomplissement de l'action (si action il y a) qui a amené le locuteur à utiliser la forme 
surcomposée. Nous avons plutôt l'impression qu'il se sert de cette forme verbale car elle lui permet de mettre en relief encore une fois la vérité de l'événement, donc la participation aux fêtes pour les enfants, dont il a déjà parlé auparavant. Notre deuxième exemple nous confortera dans cette interprétation.

Exemple 8 - Dialecte : bavarois du nord ; le locuteur n'utilise pas le «Plusquamperfekt» :

"Auf einmal bin ich aber da gesessen auf dem Arsch, hat's mich erwischt..$^{(1)}$ Von dreihundertachtzig Volt, wenn die einen erwischen, sind ja wohl bloß fünfzehn Ampere Abzieher gewesen, aber mir langt's, ich habe eine halbe Stunde geschwitzt. Na hat mich das erwischt gehabt, ${ }^{(2)}$ hat mich hingehauen. " "Tout d'un coup, j'étais assis sur les fesses, là, ça m’a frappé, ${ }^{(1)}$ (le locuteur s'est pris une décharge électrique) 380 volts, quand tu te les prends, je crois qu'il s'agissait de seulement 15 ampères, mais moi, ça me suffit, j'ai transpiré pendant une demi-heure. Alors là, ça m’a frappé, ${ }^{(2)}$ ça m’a bouleversé. "

(1) Hat's mich erwischt.

A ça me frappé.

(2) $\mathrm{Na}$ hat mich das erwischt gehabt.

Alors là a me ça frappé eu.

Le verbe allemand " erwischen ", ici traduit par "frapper ", fait partie des verbes ponctuels qui caractérisent des actions brusques. La notion d'une quelconque durée est exclue pour cette catégorie de verbes de par leur sémantique inhérente. Deux aspects nous semblent particulièrement intéressants ici. Comme pour l'exemple précédent, le locuteur reprend exactement la même formulation en la transformant au surcomposé. Immédiatement après, il continue au Perfekt. Puisqu'il s'agit d'un événement très brusque, il est difficile de l'illustrer en détail ou au ralenti. Le locuteur donne alors des explications complémentaires au Präsens (présent) quand il parle de vérités générales ("von dreihundertachtzig Volt, wenn die einen erwischen ") ou bien au "Perfekt» pour illustrer les conséquences de l'impact de la charge électrique ("ich habe eine halbe Stunde geschwitzt ", " hat mich hingehauen »). Sauf quand il revient sur l'événement lui-même, qui l'a sûrement profondément ébranlé, il se sert d'un surcomposé. L'effet, pour nous, est la mise en relief de cette expérience extrêmement courte et douloureuse, comme pour dire : "Effectivement, ça m’a carrément frappé ! »Comme pour l'exemple précédent, une interprétation qui se base sur la capacité d'un surcomposé en allemand à souligner l'aspect perfectif d'un événement nous semble peu justifiée, simplement parce qu'une autre lecture du verbe au Perfekt simple que celle d'un accompli n'est guère possible.

Notre troisième exemple se distingue des deux autres dans la mesure où la reprise au surcomposé de la phrase à l'origine au Perfekt simple contient des éléments qui pourraient très bien apporter des explications à l'emploi du surcomposé : 
Exemple 9 - Dialecte : bas-allemand (est), Poméranien (est), le locuteur utilise le "Plusquamperfekt» :

S1: Was haben denn die Pommern am liebsten gegessen zum Mittag, äh Zusammengekochtes, oder so. S2: Ach äh das kann ich gar nicht sagen, will mal sagen, ja S1: Fleisch und Gemüse und Kartoffeln so zusammen, oder? S2: \a, wir haben durcheinander gekocht, ${ }^{(1)}$ wir haben auch so jedes für sich gekocht gehabt. ${ }^{(2)} \mathrm{Daß}$ so so Spezialgericht.

S1 : Les Poméraniens, qu'ont-ils préféré comme déjeuner : des choses cuisinées, tout mélangé, par exemple ? S2 : Ben je ne peux pas vous dire ça, je dirais que oui. $S 1$ : De la viande, des légumes, des pommes de terre, tout mélangé, non ? S2 : Oui, nous avons cuisiné tout mélangé, ${ }^{(1)}$ nous avons aussi cuisiné chacun des ingrédients séparément, ${ }^{(2)}$ des repas spéciaux, quoi.

(1) Ja, wir haben alles durcheinander gekocht.

Oui, nous avons tout mélangé cuisiné.

(2) Wir haben auch so jedes für sich gekocht gehabt.

Nous avons aussi chacun desingrédients séparément cuisiné eu.

Après avoir répondu à la question de l'intervieweur en disant qu'ils ont effectivement cuisiné tous les ingrédients mélangés, réponse au "Perfekt », le locuteur modifie sa réponse en rajoutant qu'il leur est arrivé aussi de cuisiner autrement, c'est-à-dire de cuisiner chacun des ingrédients séparément. Il ne s'agit pas ici seulement d'une rectification car, comme le dit le locuteur lui-même, c'étaient bien des repas "spéciaux" qui nécessitaient une manière de cuisiner différente de celle qu'ils utilisaient d'habitude. Il semble que ce soit la particularité de ces repas qui amène le locuteur à employer une autre forme verbale que celle qu'il utilisait pour la préparation de plats normaux. Dans cet exemple, où la reprise des propos au surcomposé suit immédiatement celle qui contient la forme du Perfekt simple, il serait difficile de penser à une autre explication, pour l'apparition du double Perfekt, que celle que nous proposons. Les deux événements ont eu lieu dans la même période et sont présentés comme accomplis. Au niveau phonétique, cet exemple est également intéressant en ce qui concerne l'intonation. Dans la phrase qui contient le double Perfekt, nous remarquons d'abord un accent sur le mot «sich », qui exprime le contraire du "tout mélangé " de la phrase précédente, suivi d'une très longue pause avant les deux formes de Partizip pour que cette information nouvelle et surprenante puisse faire son effet chez l'intervieweur.

\section{Le double Perfekt inaccompli}

Si le double Perfekt en allemand sert à souligner l'aspect perfectif d'une action, on peut s'attendre à ce que cette forme verbale soit incompatible avec la notion d'une durée ou bien avec des contextes qui expriment une habitude. Or, comme le montreront les exemples suivants, on trouve bien des cas où l'un comme l'autre est possible. 
Notre premier exemple est emprunté à Rödel (2006). ${ }^{25}$ Il présente la phrase suivante comme preuve de la nature accomplie des formes surcomposées. Or, en regardant d'un peu plus près, nous allons voir qu'en réalité, c'est tout le contraire. Il s'agit ici du propos d'un joueur de football qui parle de sa passion pour ce sport, qui dure depuis très longtemps déjà.

Exemple 10 :

"Ich spiele leidenschaftlich gerne Fußball, habe es auch damals schon gespielt gehabt. "

Je joue passionnément aufoot ai $y$ aussi autrefois déjà joué eu.

" J'adore jouer au football, à cette époque j’y jouais déjà aussi. "

Laspect inaccompli de cette remarque devient évident dans la première partie de cette phrase. Comme le joueur le dit lui-même, sa passion pour le foot ne s'est jamais arrêtée. À l'époque comme aujourd'hui, il a joué au foot. À aucun moment, il n'y eut d'interruption. Pour Rödel (2006), il n'y a qu'une seule interprétation "pertinente " de cette phrase, à savoir l'intention du locuteur de présenter l'événement d' "y avoir déjà joué autrefois " comme perfectif, vu dans son ensemble. ${ }^{26}$ Mais pourquoi, faut-il se demander, le locuteur présenterait-il cet événement comme accompli si c'est justement son intention de nous parler de sa passion pour le foot qui n'a jamais connu d'interruption ? Cela ne nécessiterait-il pas beaucoup moins de suppositions si l'on convenait que ce surcomposé permet de mettre en relief le fait que cette passion pour le foot remonte déjà à l'enfance ? C'est en tout cas l'interprétation que nous privilégions.

Nous avons non seulement ici un exemple évident de l'emploi d'un surcomposé dans un contexte clairement inaccompli. Du point de vue contrastif, nous constatons ici également une grande différence avec le français. Car les caractéristiques du passé surcomposé qui sont les suivantes : un événement définitivement accompli, dont il est improbable qu'il se reproduise, inscrit dans un passé lointain avec une localisation temporelle indéfinie, excluent que l'éventualité soit encore valable au moment de l'énonciation. Tandis qu'il est impossible en français de continuer une proposition au passé surcomposé en disant "... et continue à le faire ", ceci semble être tout-à-fait possible en allemand. L'exemple suivant confirme cette observation. Le locuteur décrit la découverte d'un lapin mort sous la neige qui s'y trouve depuis un certain temps. Le locuteur utilise ici un double "Plusquamperfekt».

Exemple 11 - Dialecte : Allemagne centrale (est), silésien ; le locuteur utilise le «Plusquamperfekt » : 
" Nun hatte er schon eine Zeit gelegen dort gehabt und da kam's ein bißchen gelb durch den Schnee durch. (...)»

" Il (le lapin) y avait été depuis un certain temps et on apercevait du jaune à travers la neige. "

Nun hatte er schon eine Zeit gelegen dort gehabt. avaitil déjà un certain temps été y eu.

Le locuteur nous informe qu’à son arrivée - à un moment dans le passé -, le lapin mort se trouve déjà à cet endroit depuis quelque temps. L'important c'est que l'éventualité « d'être mort sous la neige » inclut le moment de la découverte par le locuteur et va probablement même au-delà. Le verbe principal « liegen » (littéralement être couché ou allongé) étant un verbe statique qui ne provoque pas de changement d'état, il dispose de par sa sémantique un aspect inhérent imperfectif. De ce fait, il faudrait donc avancer beaucoup d'hypothèses supplémentaires si l'on voulait voir dans cette phrase l'exemple d'un accompli.

Notre dernier exemple, trouvé sur un forum Internet, dont l'orthographe révèle une grande proximité avec le langage parlé, présente un autre exemple illustrant l'emploi d'un surcomposé dans un contexte inaccompli. Le locuteur fait référence au cas d'Anders Breivik, le tueur norvégien de 2011. Il répond à un autre internaute en lui expliquant qu'il ne s'agit pas d'un soudain changement d'opinion de la part des autorités judiciaires norvégiennes quand cellesci refusent à l'accusé de témoigner en public.

Exemple 12 :

"Nene, das ham se doch von Anfang an gesagt gehabt. Sie wollen ihm keine öffentliche Plattform bieten (...)»

«Non non, elles (les autorités judicaires) l'ont dit depuis le début. Elles ne veulent pas lui offrir une tribune."

Das ham se doch von Anfang an gesagt gehabt.

Ça ontelles depuisle début dit eu.

L’emploi de l'adverbial "depuis le début " situe l'éventualité de manière à ce qu'elle inclue le moment de l'énonciation. Car les autorités judiciaires norvégiennes ne veulent toujours pas que l'accusé se prononce en public, comme elles l'ont dit depuis le début de cette affaire. Comme nous l'avons déjà vu dans l'exemple 4, il est possible de continuer la partie au surcomposé " das ham se doch von Anfang an gesagt gehabt " en disant "... et ils continuent à le faire».

Avec cette dernière phrase, qu'on entend souvent en langage parlé, nous avons désormais suffisamment d'exemples pour montrer que, contrairement au français et à l'encontre de la définition de l'aspect accompli, les formes surcomposées allemandes peuvent bel et bien inclure le moment de l'énonciation. Dans ce qui suit, nous allons voir qu'elles sont même capables d'exprimer des habitudes du passé, traditionnellement associées avec l'aspect inaccompli, un emploi 
qui est " par définition " exclu pour les formes surcomposées françaises. ${ }^{27}$ En tant que véritables formes exprimant un accompli les surcomposés régionaux français désignent une action qui se déroule à un ou plusieurs moments non spécifiques dans le passé, et dénotent donc une action non habituelle. Dans l'exemple 13, le locuteur, auquel l'intervieweur demande d'expliquer comment on préparait la choucroute autrefois, décrit la procédure en mettant l'accent sur le fait qu’à un moment donné il fallait emprunter une râpe à choucroute.

Exemple 13 - Dialecte : allemand central (est), silésien, le locuteur utilise le « Plusquamperfekt " :

S1: Erzählen Sie mal, wie das gemacht wird! S2: Nun, das Sauerkraut! Da hatten wir ein Krauttönnchen, das wurde natürlich schon, ein paar Tage davor wurd's ausgewaschen, und dann haben wir halt den Krauthobel immer gewöhnlich geborgt gehabt, und da wurde halt das Krautköpfl, das wurde in Viertel geschnitten.

S1 : Racontez comme ça se fait. S2 : Alors, la choucroute. Nous avions un petit récipient pour le chou qui avait bien sûr été nettoyé quelques jours avant déjà et puis nous avions toujours l'habitude d'emprunter la râpe à choucroute et après on coupait le chou en quatre.

Dann haben wirhalt den Krauthobel immer gewöhnlich geborgt gehabt.

Puis avons nous larâpeàchoucroute toujours d'habitude emprunté eu.

Cet exemple est intéressant pour plusieurs raisons. En lisant ce récit, il nous semble très probable que les étapes sont présentées dans leur ordre chronologique, si bien que le nettoyage du récipient précède l'emprunt de la râpe à choucroute qui est suivi du coupage des choux. Le locuteur utilise la forme surcomposée au milieu d'une narration avec l'adverbe de temps " dann " (puis). Nous avons donc ici un autre exemple qui tend à montrer que tous les surcomposés en allemand ne sont pas des antérieurs d'un antérieur. Deuxièmement, dans une énumération d'actions nécessaires pour la fabrication de la choucroute, seul l'emprunt de la râpe apparaît à la voix active (ici au double Perfekt) tandis que les autres actions nous sont racontées à la forme passive. Ce faisant, le locuteur reprend donc la forme verbale impersonnelle que l'intervieweur a utilisée dans sa question. ("Erzählen Sie doch mal, wie das gemacht wird! ") Bien sûr, le passif avec "werden » en allemand présente la situation en tant que processus. Néanmoins, il nous semble que le choix du double Perfekt pour l'emprunt de la râpe à choucroute ne se justifie pas par la mise en avant de l'accomplissement de cette action. Tout simplement parce que l'action consistant à emprunter ne peut pas facilement être présentée comme processuelle. Pour nous, il s'agit plutôt de la mise en relief d'une information importante et ceci aux trois niveaux linguistiques. Le changement de voix indique que l'emprunt de la râpe à choucroute

27 Cf. Carruthers Janice, 1994, p. 179. 
ne fait pas partie des étapes normales de la fabrication, parce que le locuteur utilise le pronom personnel "nous ". Pour les étapes suivantes il reprend le passif. Cette interprétation est confortée par une autre observation cette fois au niveau phonétique. Dans le cas présent, l'intonation de la phrase contenant la forme surcomposée montre une évolution assez remarquable dans la mesure où le locuteur met un accent fort sur le mot "geborgt " (emprunté), plus précisément sur le radical «borgt ». Il semble que l'information la plus importante que veut véhiculer le locuteur est le fait qu'il a fallu emprunter la râpe à choucroute. En revanche, le besoin d'une râpe à choucroute est en soi tellement évident pour le locuteur qu'il considère inutile de le mettre en avant. De ce point de vue, l'emploi du double Perfekt semble être justifié en tant que moyen supplémentaire afin de mettre en relief une information considérée comme importante. Sous l'angle de l'aspectualité, l'observation la plus intéressante est pourtant que le locuteur utilise deux adverbes qui montrent que l'emprunt de la râpe à choucroute représentait, dans son cas, une action habituelle dans le passé : "immer " (toujours) et "gewöhnlich» (d'habitude).

L'exemple suivant, qui est très récent, montre lui aussi l'emploi d'une forme surcomposée pour parler d'une action habituelle dans le passé. La Ministre-Présidente de la Rhénanie-du-Nord-Westphalie, Hannelore Kraft, parle lors d'une conférence de presse du soutien que lui avait apporté son entourage pendant la campagne électorale.

Exemple 14:

"Mama hat immer alle Sachen richtig gebügelt gehabt. Das ist auch nicht zu unterschätzen. $»^{28}$

" Maman a toujours bien repassé toutes mes affaires. Ceci n'est pas à sousestimer non plus. »

Mama hat immer alle Sachen richtig gebügelt gehabt. Maman a toujourstoutes les affaires bien repassé eu.

L'aide régulière ( toujours») de sa mère, aussi petite qu' elle semble, mérite pour Mme Kraft non seulement d'être mentionnée. Elle souligne l'importance de cet événement, à première vue sans intérêt, en utilisant la forme d'un double Perfekt et en qualifiant la valeur de ce soutien de précieux.

\section{Conclusion}

L'analyse à partir de nos exemples d'une petite partie des formes verbales surcomposées en allemand et en français nous a fourni suffisamment d'indices pour pouvoir remettre en question une interprétation purement aspectotemporelle de ces formes. En français comme en allemand, les valeurs tempo- 
relles ou aspectuelles des formes analysées dans cet article coïncident avec celles d'autres formes verbales, notamment le passé composé ou bien le Perfekt.

Dans cet article, nous avons proposé pour l'allemand et le français un nouvel élément descriptif des formes, en liaison avec la fonction expressive. Il consiste en une mise en relief de l'action verbale qui souligne le fait qu'un événement "s'est vraiment passé comme cela ». Au moins pour l'allemand, nous attribuons cette expressivité en partie à la valeur de "vérité " qu'apporte le participe II de l'auxiliaire. En français, cette valeur ne se montre pas avec la même clarté qu'en allemand. Son existence ne peut pourtant pas être exclue, mais il faut plus de recherches pour trancher cette question.

Quoi qu'il en soit, les formes verbales surcomposées nous rappellent une fois de plus que nous échouons souvent dans la tentative de restreindre la fonction d'une forme verbale à une seule valeur.

\author{
Norman Hass \\ EA 4593 CLARE \\ Université Bordeaux Montaigne \\ norman.hass@u-bordeaux-montaigne.fr
}

\begin{abstract}
Résumé
Le français et l'allemand connaissent des formes verbales rares que la linguistique appelle formes surcomposées. Dans les deux langues elles consistent en une forme de l'auxiliaire avoir (haben) ou être (sein), du participe passé (Partizip II) du verbe principal et du participe passé (Partizip II) du même auxiliaire. Pour les deux langues, la description de leur valeur sémantique pose beaucoup de problèmes. Pour le français, il a déjà été démontré que les formes en question pourraient prendre des fonctions qui dépassent les approches classiques aspecto-temporelles. On évoque notamment une fonction expressive qui aurait pour effet une interprétation des énoncés au passé surcomposé comme étant " exceptionnels " ou " très personnels ". À partir d'un grand nombre d'occurrences des formes surcomposées de l'allemand parlé, nous allons voir que cette même approche pourra servir ici d'explication.
\end{abstract}

\title{
Abstract
}

Though relatively rare, double Perfect constructions exist in French (passé surcomposé) as well in German (doppeltes Perfekt). In both languages, they are composed of an auxiliary "have" or "be" (haben/avoir or sein/être) and the past participle of the main verb. In addition, they take the past participle of the auxiliary (gehabtleu or gewesen/été). The semantic description of their meaning poses huge problems. For French, it has already been shown that double Perfects can have functions which exceed the classical temporal and aspectual approaches. It is their expressive function which leads to the frequent interpretations of utterances in a double Perfect as "exceptional" or "bighly personal". On the basis of a number of examples of double Perfects in spoken German, we will try to show that the same interpretation could probably hold true here, too. 


\section{ANNEXE \\ Liste des occurrences allemandes issues du Corpus « Gesprochenes Deutsch» (IdS Mannheim)}

\section{Exemple 4}

„Da konnten wir ganz auf den Bodensee rübergucken, so in die Schweiz rein. Und mit dem Fernglas, wir konnten auch so ungefähr sehen, daß oben so in den Bergen sogar noch Schnee lag. Also, das war ganz prima. Uns hat das ganz prima gefallen. $\mathrm{Na}$ ja, so um acht, halb neun, neun, genau weiß ich das nun auch nicht mehr, sind wir dann (PAUSE) da haben wir des Morgens erst mal was gegessen gehabt, und dann sind wir dann um neun Uhr losgefahren wieder."

\section{Exemple 5}

„Aber Mutter, die wollte das doch nicht so ganz glauben, ich lag schon im Bett. Und dann nachher am nächsten Tag, dann hat sie mich natürlich vorgekriegt gehabt, hat gesagt: „Junge, willst du es jetzt noch bestreiten?" $\mathrm{Na}$, lügen wollte ich nicht, und dann habe ich gesagt: „Ja, Mutter, ich habe das Bettlaken umgehabt, und ihr beide Frauen hattet mehr Angst, als Vaterlandsliebe!"

\section{Exemple 6}

„(...) zu Mittag hat's dann wieder die Frau sein Kind rausgeschickt, und dann sind wieder alle Bohnenkerne weg gewesen, die Vögel und Tauben haben sie alle gefressen gehabt, dann ist es wieder als tiefer in ' Wald reingelaufen und tiefer, und hat (PAUSE) und dann ist es wieder Abend geworden und dunkel und die Eulen haben wieder runtergeguckt, (...)

\section{Exemple 7}

S1: Haben Sie dann auch mal ein Kinderfest in der Schule so mitgemacht? S2: Ja, Kinderfest habe ich ja in mein ersten Jahren ja mitgemacht, weil das nachher in den Kriegsjahren wurde das ja abgesagt, da waren ja keine öffentliche Veranstaltungen irgendwie. $\underline{I n}$, in den ersten Jahren habe ich das ja mitgemacht gehabt. Dann war ja Topfschlagen und Fischstechen und Eierlaufen, alles so was mußte man ja, nicht, und.

\section{Exemple 8}

„Auf einmal bin ich aber da gesessen auf dem Arsch, hat's mich erwischt. Von dreihundertachtzig Volt, wenn die einen erwischen, sind ja wohl bloß fünfzehn Ampere Abzieher gewesen, aber mir langt's, ich habe eine halbe Stunde geschwitzt. Na hat mich das erwischt gehabt, hat mich hingehauen."

\section{Exemple 9}

S1: Was haben denn die Pommern am liebsten gegessen zum Mittag, äh Zusammengekochtes, oder so. S2: Ach äh das kann ich gar nicht sagen, will mal sagen, ja S1: Fleisch und Gemüse und Kartoffeln so zusammen, oder? S2: Ja, wir haben durcheinander gekocht, wir haben auch so jedes für sich gekocht gehabt. Daß so so Spezialgericht.

\section{Exemple 11}

„Ich dachte, verflucht noch einmal, der kann uns doch nicht alle hier zum Narren haben, der Hase muß ja hier wo sein, nicht! Es waren ein paar kleine Tümpel drin, und da war so Eis drauf, und der Schnee lag etwas drauf, und dann stand ich so da, da denke ich, hier ist so eine, ist mit dem Fuß gescharrt, und da hat der den Hasen an den Rand gelegt vom Lehm und hat mit einem, mit dem Fuß den Schnee drübergemacht. Nun hatte er schon eine Zeit gelegen dort gehabt und da kam's ein bißchen gelb durch den Schnee durch. (...)“ 


\section{Exemple 13}

S1: Erzählen Sie mal, wie das gemacht wird! S2: Nun, das Sauerkraut! Da hatten wir ein Krauttönnchen, das wurde natürlich schon, ein paar Tage davor wurd's ausgewaschen, und dann haben wir halt den Krauthobel immer gewöhnlich geborgt gehabt, und da wurde halt das Krautköpfl, das wurde in Viertel geschnitten, und da hatten wir so ein Kästchen drauf, wurde ein Kopf reingequetscht, und da haben wir das Kraut gehobelt. (...)

Exemple 14 (trouvé dans le journal hebdomadaire allemand „Die Zeit")

„Mama hat immer alle Sachen richtig gebügelt gehabt. Das ist auch nicht zu unterschätzen.“ 